\title{
Comparison of fast tomographic methods for application on the Soft X-Ray Tomography System on Wendelstein 7-X stellarator
}

\author{
P. J. Carvalho*, H. Thomsen ${ }^{\dagger}$, S. Gori**, U. v. Toussaint**, J. Geiger ${ }^{\dagger}$, A. Weller ${ }^{\dagger}$, R. \\ Coelho* and H. Fernandes* \\ *Associação Euratom/IST Centro de Fusão Nuclear, Instituto Superior Técnico 1049-001 Lisboa, Portugal \\ ${ }^{\dagger}$ Max-Planck-Institut fur Plasma Physik, EURATOM Assoziation, 17491 Greifswald, Germany \\ ${ }^{* *}$ Max-Planck-Institut für Plasma Physik, EURATOMAssoziation, 85748 Garching, Germany
}

\begin{abstract}
The Wendelstein 7-X stellarator, presently under construction in Greifswald, is foreseen to operate on a steady state regime. Under such a scenario, a constant diagnosis of the plasma characteristics is strongly envisaged. A X-Ray Tomography diagnostic is a particularly useful tool since a poloidal cross-section of the plasma's X-Ray emissivity can be reconstructed and the plasma's position as well as MHD activity can be inferred.

Fast tomographic algorithms such as the Cormack inversion or neural networks (NN) can be applied to obtain reconstructions at a human time scale $(10 \sim 100$ reconstructions per second). This paper discusses the potential application of these algorithms on the Wendelstein 7-X stellarator by comparing performance and reliability of the results. The NN reconstruction has proven to be faster and more reliable than the Cormack's.
\end{abstract}

Keywords: Wendelstein 7-X, Stellerator, X-Ray, XMCTS, Tomography, Fourier-Bessel, Neural-Networks, reconstruction, inversion PACS: $52.55 . \mathrm{Hc}, 81.70 . \mathrm{Tx}$

\section{INTRODUCTION}

The Wendelstein 7-X stellarator is foreseen to operate on a steady state regime. Such a scenario requires various on-line plasma diagnostics. One diagnostic is the X-Ray Multi Camera Tomography System (XMCTS) which can reconstruct a poloidal cross-section of the plasma's X-Ray emissivity and infer the plasma position and MHD mode activity.

This paper presents two different tomographic algorithms which can be processed in a human time scale (1 reconstruction in $10 \mathrm{~ms}$ ) using, for example, a real-time operating system in a standard PC. The algorithms are the Fourier-Bessel [1,2] and a Neural-Network (NN) method [3]. The Fourier-Bessel algorithm uses a fit of the available data to a set of basis functions: the Bessel functions are used in radial direction and the Fourier sine and cosine components for the poloidal direction. The $\mathrm{NN}$ is trained with phantom data to produce a given output for some predefined inputs. It will produce a good reconstruction if the analyzed data is an interpolation of the training set.

The XMCTS design consists of 20 cameras, each with 18 active channels. Their positions and sightlines are sketched in fig. 1 [4].

\section{ALGORITHMS}

In tomography, the goal is to generate a cross-section view of a given substance from a set of integrated measurements.

Of all the tomographic algorithms tipically used by the fusion community, the most appropriate for real-time application are Cormack based methods and Neural-Networks, because the other methods are usually iterative and the convergence time is unknown.

The Cormack method [5] essentially approximates the emissivity profile by a Fourier expansion on the poloidal plane and an expansion in Zernicke polynomials in the radial direction. However, Zernicke polynomials have the inherent problem that they are non zero at the outer edge which can lead to completely unrealistic results. So a different type of functions must be used in the radial expansion and Wang [1,2] found that the first order Bessel functions are appropriate. 


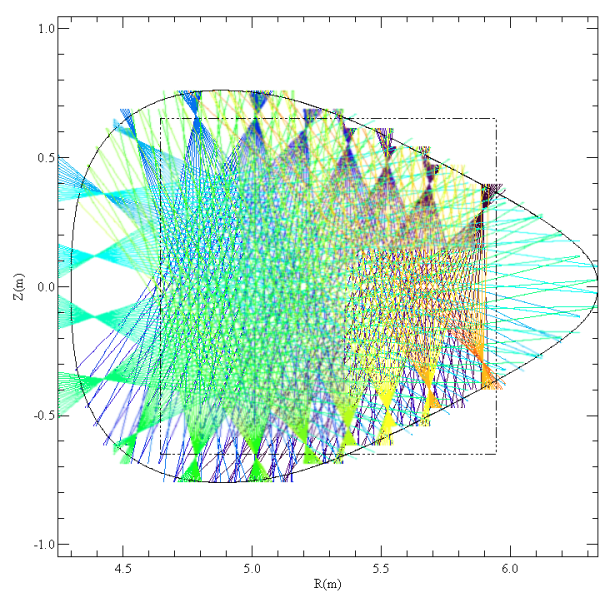

FIGURE 1. The tomography camera system of the Wendelstein 7-X stellerator. There are 20 linear pinhole cameras with 18 active channels, each. The square shows the position of the area where the reconstructions are calculated.

The test radiation distributions (phantoms) used in this work were generated using a radial gaussian profile overlapped on equilibria flux surfaces. The VMEC algorithm [6] was used to generate these flux surfaces for different magnetic configurations of $\mathrm{W} 7-\mathrm{X}$.

\section{Fourier-Bessel basis functions}

This algorithm is described in detail elsewhere $[1,2,5]$. In this work, the same nomenclature as in ref. [2] is used.

The Radon transform in two dimensions can be written as $f(p, \phi)=\int_{L(p, \phi)} g(r, \theta) d s$ where the integral path $L(p, \phi)$ is a line of sight which traverses the plasma, $g(r, \theta)$ is the plasma emissivity in poloidal coordinates and $f(p, \phi)$ is the measurement along the line $L$. Fourier expanding these two functions one can write them as

$$
\begin{aligned}
& g(r, \theta)=\sum_{m=0}^{\infty}\left(\sum_{l=0}^{\infty}\left(a_{m l}^{c} g_{m l}(r)\right) \cos (m \theta)+\sum_{l=0}^{\infty}\left(a_{m l}^{s} g_{m l}(r)\right) \sin (m \theta)\right) \\
& f(p, \phi)=\sum_{m=0}^{\infty}\left(\sum_{l=0}^{\infty}\left(a_{m l}^{c} f_{m l}(p)\right) \cos (m \phi)+\sum_{l=0}^{\infty}\left(a_{m l}^{s} f_{m l}(p)\right) \sin (m \phi)\right)
\end{aligned}
$$

It is impractical to follow these summations to infinity, so they must be truncated. This trucation is done according to the following criteria: The $l$ index is associated with the radial component of the expansion. The resolution of this component is determined by the number of photodiodes in each camera, according to the Nyquist theorem; in the present case of 18 photodiodes per camera, $N_{l}=9$, or $l=0,1, \cdots, 8$. The $m$ index is associated with the angular component of the expansion and its resolution is determined by the number of cameras. For 20 equidistant cameras, one can have up to $m=9$, but W7-X's cameras are not equidistant so one must use less than that. Trial and error yields best results for $N_{m}=7$, or $m=0,1, \cdots, 6$.

If all $a_{m l}^{c, s}$ s are known, one can use eq. (1) to generate the tomographic reconstruction. Each photodiode's position is given by $\left(p_{i}, \phi_{i}\right)$, so one can write $f_{i}=f\left(p_{i}, \phi_{i}\right)$. By writing $X_{i, m l}^{c}=f_{m l}\left(p_{i}\right) \cos \left(m \phi_{i}\right)$ and $X_{i, m l}^{s}=f_{m l}\left(p_{i}\right) \sin \left(m \phi_{i}\right)$ one can use the matrix notation $\vec{f}=\mathbf{X} \cdot \vec{a}$. Since there are more equations than unknowns, $\mathbf{X}$ is not a square matrix so a simple matrix inversion is not possible. A least squares fit can be performed using the Singular Value Decomposition (SVD) of the matrix $\mathbf{X}=\mathbf{U} \mathbf{W V}^{\mathrm{T}}$ [7] with $\vec{a}=\sum_{i=0}^{M} \frac{\vec{U}_{i} \cdot \vec{f}}{W_{i}} \cdot \vec{V}_{i}$, where $\vec{U}_{i}\left(\vec{V}_{i}\right)$ is the $i$ 'th column of the matrix $\mathbf{U}(\mathbf{V}), W_{i}$ is the $i$ 'th diagonal value of the matrix $\mathbf{W}$ and $M$ is the number of columns of $\mathbf{X}$. Since $\mathbf{X}=\mathbf{X}(p, \phi)$, it can be precalculated as well as it's SVD. Thus, for each tomogram, only $\vec{a}$ needs to be calculated and the reconstruction can then be built on any $(x, y)$ grid. In this case, a $30 \times 30$ grid was used. It doesn't take advantage of the full resolution of the system, but it can give a good result in a fast time scale. 


\title{
Neural Networks
}

A Neural-Network (NN) consists of simple processing units (neurons) connected amongst each other in a particular way. Typically, a NN is arranged in layers of neurons, where any one neuron can have a connection to all other neurons on the previous layer. Each neuron has one activation function which can be any function of neurons from the previous layer(s). The activation function $z$ used in this work is $z=\tanh \left(\sum_{i} w_{i} a_{i}\right)$, where $a_{i}$ is the output from the neuron $i$ of the previous layer and $w_{i}$ is the weight that neuron $i$ has on the present neuron.

There are many types of NNs. In this work, a multi-layer perceptron was used. A NN must be trained in order to determine the weights. This training is done with an artificial input and output data set. For tomography, one generates a set of phantoms of expected spacial radiation distributions of the plasma and, using virtual sensors on this phantom, generates the corresponding sensor data.

The perceptron networks have one extra neuron in each layer which isn't related to the previous layers; this is the bias neuron and is usually set to 1 . The training and layer optimization of the network was performed using bayesian methods [3] and produced a two hidden layer network where the first hidden layer has 9 neurons and the second hidden layer has 11 neurons. The inputs for the network are the data channels from the photodiodes and the $(x, y)$ position of the pixel being reconstructed and the output is the value of this pixel. So for each reconstruction, the NN must run for as many times as there are pixels in the reconstruction grid $(40 \times 30=1200$ runs).

\begin{abstract}
ANALYSIS
The algorithm speed tests were performed on a standard PC running Windows ${ }^{\circledR X P}$, with an Intel ${ }^{\circledR}$ Centrino ${ }^{\mathrm{TM}}$ processor at a clock speed of $1.6 \mathrm{GHz}$, using the IDL programming language [8]. The marks achieved here can be drastically improved by using the $\mathrm{C}$ programming language, for example, and running it under a real-time operating system, as well as using a faster processor.

For this work, not all channels are used as inputs to the Neural Network because it is possible to have a satisfying reconstruction using a subset of the channels. Instead of using all 360 channels, only 60 were used, one for each 6 consecutive channels.

The maximum order of the Fourier-Bessel decomposition determines the resolution of structures in the reconstruction. But, the higher the order, the slower the algorithm is and more artefacts are introduced, compromising the reliability of the reconstruction. In this work, a maximum $m=6$ and a maximum $l=6$ were used.

The circle where the Fourier-Bessel reconstruction is built lies inside a square $30 \times 30$ grid and it takes about $280 \mathrm{~ms}$ to produce one reconstruction. The Neural Network algorithm takes about $40 \mathrm{~ms}$ for the whole $40 \times 30$ grid. A dummy $\mathrm{NN}$ was used to test the duration of the algorithm if all 360 channels were used and it created the reconstruction in about $100 \mathrm{~ms}$.

One of the potential uses of the tomography system is to determine the plasma's $\beta$ value. The relation between $\beta$ and $R$ is shown in fig. 2 a). The NN follows this relation closely, while the FB results are less accurate for low $\beta$ s. Figure 2 b) shows 3 reconstructions from those contemplated in the chart in fig. 2 a). It can be seen that the NN algorithm is much more accurate than the FB.

The errors presented were determined with the formula $\varepsilon=\frac{\sum_{x, y}\left(g_{p h}(x, y)-\hat{g}(x, y)\right)^{2}}{\sum_{x, y}\left(g_{p h}(x, y)\right)^{2}}$, where $g_{p h}$ is the phantom and $\hat{g}$ is the reconstruction.

\section{DISCUSSION}

The Fourier-Bessel algorithm took 7 times as much time as the Neural Network, for the same setup. The NN takes $40 \mathrm{~ms}$ to produce one reconstruction and it can be much faster on a different system, thus fulfilling the human timescale requirement. The NN has shown to produce very good reconstructions, but only if the data used is an interpolation of the trained data. When required to extrapolate on the trained data, the NN struggles, altough the result is never as bad as those from the FB algorithm. The FB algorithm uses circular base functions, making it challenging to reconstruct a triangular shape. Only the central part of the reconstruction is good, but the peripheral part is solely composed of artifacts created by the overlapping of the basis functions.

It has been shown that it is possible to determine the $\beta$ of the plasma using the tomographic reconstruction via the NN. However, if the goal is to determine $\beta$, one can train a NN solely for this purpose using even fewer channels of
\end{abstract}




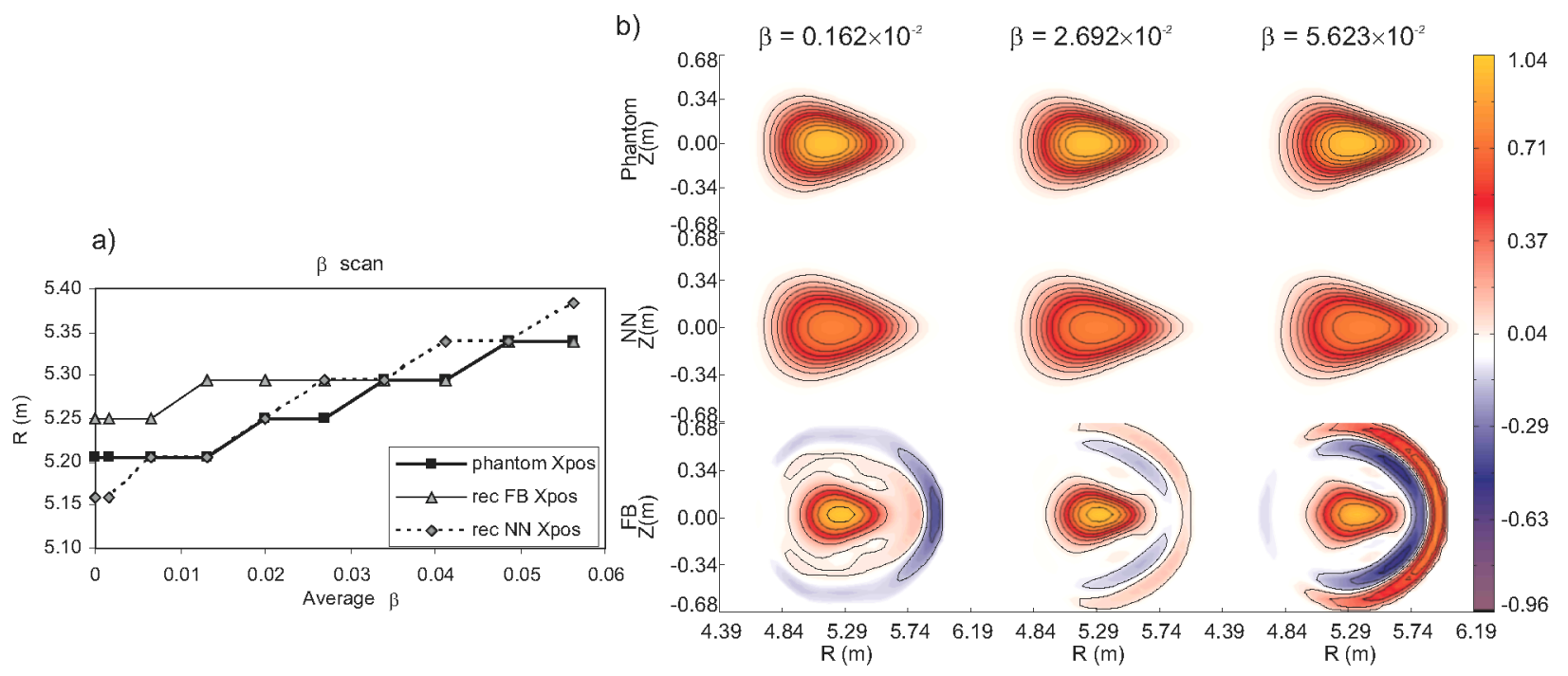

FIGURE 2. The chart a) compares the position of the maximum of the phantom and the reconstructions using Fourier-Bessel (FB) and Neural Networks (NN) algorithms for the W7X configuration 1000_1000_1000_1000_+0000_+0000 and increasing $\beta$ values. Each $5 \mathrm{~cm}$ jump in the $R$ axis is due to a one pixel difference in the reconstruction grid. The $\overline{N N}^{-} \bar{a}$ a better agreement with the phantoms and this can be seen in the reconstructions b). The artifacts arising from the circular nature of the FB algorithm are evident altough the central parts show some resemblance with the phantoms.

\section{the XMCT System.}

One potential application of the FB algorithm is to check if the center area of the reconstruction is consistent with the NN, because the FB is more generic and not bound by training.

\section{ACKNOWLEDGMENTS}

This work has been carried out within the framework of the Contract of Association between the European Atomic Energy Community and "Instituto Superior Técnico". Financial support was also received from "Fundação para a Ciência e Tecnologia" in the frame of the Contract of Associated Laboratory.

\section{REFERENCES}

1. Ling Wang and Robert S. Granetz. A simplified expression for the radon transform of bessel basis function in tomography. Review of Scientific Instruments, 62:842-843, 1991.

2. Ling Wang and Robert S. Granetz. An analytical expression for the radon transform of bessel basis function in tomography. Review of Scientific Instruments, 62:1115-1116, 1991.

3. Udo v. Toussaint et al. Invariance priors for bayesian feed forward neural networks. Neural Networks, 19:1550-1557, 2006.

4. A. Weller et al. Concepts of x-ray diagnostics for wendelstein 7-x. Review Scientific Instruments, 75:3962-3965, 2004.

5. A. M. Cormack. Representation of a function by its line integrals, with some radiological applications. Journal of Applied Physics, 35:2908, 1964.

6. S.P. Hirshman and O. Betancourt. Preconditioned descent algorithm for rapid calculations of magnetohydrodynamic equilibria. Journal of Computational Physics, 96:99-109, 1991

7. William H. Press, Saul A. Teukolsky, William T. Vetterling, and Brian P. Flannery. Numerical Recipes in C, The Art of Scientific Computing, Ch. 15.4. Cambridge University Press, second edition, 1992.

8. Interactive Data Language ITT Visual Information Solutions IDL. http://ittvis.com/idl/. 